\title{
Determination of Fetal Spinal Cord Measurement for Every Gestation Age
}

\author{
Hamna Qureshi ${ }^{1}$, Megan Beth Marine ${ }^{2}$, Nucharin Supakal ${ }^{2}$, Monica Forbes-Amrhein ${ }^{2}$ \\ ${ }^{1}$ Indiana University School of Medicine, ${ }^{2}$ Indiana University School of Medicine, Department of \\ Radiology \& Imaging Sciences
}

\section{Background and Hypothesis:}

Tethered spinal cord is challenging to identify in fetuses. If untreated, tethered spinal cord can result in neurologic weakness and sensory loss in the lower half of the body. The goal of this study is to develop a library of normal ratios of the position of the conus medullaris relative to the spinal canal from normal fetal magnetic resonance imaging (MRI) examinations at each gestation age. This will allow for early identification of tethered spinal cord and timely intervention. We hypothesize that the ratios will decrease throughout pregnancy as the level of the conus medullaris rises.

\section{Experimental Design or Project Methods:}

In this pilot study, a retrospective review of fetal MRIs from patients between 20-38 weeks gestation age were examined. Exclusion criteria included fetuses with fetal spinal or central nervous system anomalies, VACTERL, ventral wall defects, scoliosis, and sacrococcygeal teratoma. The length from the fetal obex to the conus medullaris was measured and defined as the spinal cord length. The length of the obex to the caudal thecal sac was defined as the spinal canal length. The ratio of the spinal cord length to the spinal canal length was calculated. The ratios at each gestation age were compared using ANOVA $(p<0.05)$.

\section{Results:}

A total of 225 patients were reviewed. We observed a statistically significant decreasing trend in fetal spinal ratios from $20-38$ weeks of gestation age. The average ratio at 20 weeks gestation age is 0.756 and at 38 weeks is 0.696 .

\section{Conclusion and Potential Impact:}

This retrospective pilot study of fetal MRIs will establish reference values for the ratio of spinal cord to spinal canal lengths at progressing gestation ages. Ultimately, these ratios can establish normal values for identification of tethered spinal cord during the fetal period, thereby allowing early intervention to mitigate complications. 